\title{
Acute lymphoblastic leukemia and obesity: increased energy intake or decreased physical activity?
}

\author{
H. Jansen • A. Postma • R. P. Stolk • W. A. Kamps
}

Received: 27 May 2008 / Accepted: 15 October 2008 / Published online: 7 November 2008

(C) The Author(s) 2008. This article is published with open access at Springerlink.com

\begin{abstract}
Background Obesity is a well-known problem in children with acute lymphoblastic leukemia (ALL), and it might be the result of an excess in energy intake, reduced energy expenditure, or both. The aim of this study is to describe energy intake and physical activity during treatment for ALL with intermittent dexamethasone (DEXA).

Methods Body mass index (BMI), energy intake, and physical activity were measured in 16 ALL patients on maintenance treatment and in 17 healthy controls. ALL patients were measured during ("on DEXA") and in between ("off DEXA") DEXA treatments.

Results In patients, the mean increase in BMI z-score was $1.4 \pm 1.1$. Energy intake on DEXA was higher $(2,125.9 \pm$ 476.0 vs $1,775.1 \pm 426.1 \mathrm{kcal} / 24 \mathrm{~h}, p<0.05)$ and energy intake off DEXA was lower $(1,305.0 \pm 249.4$ vs $1,775.1 \pm$ $426.1 \mathrm{kcal} / 24 \mathrm{~h}, p<0.05)$, compared to healthy controls. Physical activity on DEXA was lower compared to healthy controls $\left(30.0 \pm 3.9\right.$ vs $40.0 \pm 6.0 \mathrm{kcal} \mathrm{kg}^{-1} 24 \mathrm{~h}^{-1}, p<0.001$ and 7,303.1 $\pm 4,622.9$ vs $13,927.2 \pm 3,822.7$ steps, $p<0.05$ ). Physical activity off DEXA was not different compared to healthy controls.

Conclusion Weight gain in patients on ALL treatment might be owing to increased energy intake and decreased physical activity during treatment with DEXA.
\end{abstract}

\footnotetext{
H. Jansen $(\bowtie) \cdot$ A. Postma $\cdot$ W. A. Kamps

Department of Paediatrics, Division of Paediatric Oncology, Faculty of Medical Sciences,

University Medical Centre Groningen, University of Groningen, P.O. Box 30.001, 9700 RB Groningen, The Netherlands

e-mail: h.jansen@epi.umcg.nl

H. Jansen • R. P. Stolk

Department of Epidemiology,

University Medical Centre Groningen, University of Groningen,

Groningen, The Netherlands
}

Keywords Acute lymphoblastic leukemia .

Dexamethasone $\cdot$ Energy intake $\cdot$ Obesity $\cdot$ Physical activity

\section{Introduction}

Along with the gradually increasing survival rates in childhood acute lymphoblastic leukemia (ALL), there is growing concern about the quality of survival and the late effects of therapy. One major problem in childhood ALL is weight gain during treatment, resulting in obesity $[5,11]$. Possible underlying mechanisms are treatment with corticosteroids [12], growth hormone deficiency following cranial irradiation [7], and reduced habitual physical activity [10]. Obesity in general is caused by a sustained imbalance between energy intake and energy expenditure. Regarding the development of obesity during treatment of ALL, it is not clear whether it is due to an excess in energy intake, reduced energy expenditure, or a combination of both. The aim of this study is to describe energy intake and physical activity in ALL patients during and in between dexamethasone (DEXA) courses.

\section{Methods}

Patients were eligible if they were at least 4 years of age and if they were in the maintenance phase of treatment for childhood ALL with a regimen including intermittent DEXA, i.e., the Dutch Childhood Oncology Group protocols ALL-9, ALL-10, or ALL-relapse 1998. Dosages of DEXA were $6 \mathrm{mg} \mathrm{m}^{-2}$ day $^{-1}$ during 14 days in ALL-9, $6 \mathrm{mg} \mathrm{m}^{-2}$ day $^{-1}$ during 5 days in ALL-10 medium risk, $10 \mathrm{mg} \mathrm{m}^{-2}$ day $^{-1}$ during 14 days in the consolidation phase of ALL-10 standard risk, and 
$6 \mathrm{mg} \mathrm{m}^{-2}$ day $^{-1}$ during 7 days in ALL-relapse'98. Courses with DEXA alternated with courses without DEXA. Siblings nearest in age or friends were invited to participate as healthy controls. Participants were included if they had no coexisting conditions relevant to energy balance, e.g., diabetes mellitus or physical disability. The protocol was approved by the Ethics Committee of the University Medical Centre Groningen. Informed written consent was obtained from the parents of each child enrolled in the study and from participants from the age of 12 years.

Anthropometry and body composition Standing height, to the nearest $0.1 \mathrm{~cm}$, and weight, to the nearest $0.05 \mathrm{~kg}$, were measured. Body mass index (BMI) $\left(\mathrm{kg} / \mathrm{m}^{2}\right)$ was calculated as weight divided by height squared. BMI $\mathrm{z}$ scores were calculated as $(\mathrm{BMI}-<\mathrm{BMI}>) / \mathrm{SD},<\mathrm{BMI}>$ and $\mathrm{SD}$ being the mean BMI and standard deviation for the subject's age and sex, according to normative data from the Fourth Dutch Growth Study (1997) [6]. Patients' BMI and BMI z score at diagnosis were calculated retrospectively using medical records.

Energy intake and physical activity Energy intake and physical activity were measured, concurrently, twice over two consecutive days (i.e., overall, 4 days) "on DEXA" and twice over two consecutive days "off DEXA" in patients and over a random 4-day period in controls.

Energy intake was measured using a dietary diary filled in by the parents. Food intake data were converted to energy intake (kcal) using nutritional analysis software (Eetmeter 2002 C), The Netherlands Nutrition Centre, The Hague) based on the database of the chemical composition of foods in the Netherlands (NEVO, Netherlands Food Composition chart, 2001). Physical activity was measured by a proxy report (developed and validated for this age group by TNO Prevention and Health, Leiden, The Netherlands) and a pedometer. The parents reported all their children's activities and their durations. By multiplying the duration of the different activities by the matching intensity score, based on Ainsworth's Compendium of Physical Activities 2000, in which metabolic equivalent task values are assigned to physical activities, we estimated the physical activity [1]. In addition, we measured physical activity using a pedometer, the Digiwalker SW200 ${ }^{\circledR}$. This is a simple, inexpensive, but valid method to assess physical activity in this age group [3]. Participants wore the pedometers on their waists and registered the number of daily steps.

Data analysis BMI and BMI z scores at diagnosis vs BMI and BMI $z$ score at the time of the study and mean daily energy intake and physical activity on DEXA vs off DEXA were analyzed with the paired-sample $t$ test. Differences between patients and controls were analyzed with analysis of covariance to adjust for age and gender. Difference in mean BMI z score between patients and controls was tested by Student's $t$ test. $p$ Values less than 0.05 were considered statistically significant. All the analyses were carried out with SPSS 12.0.2 for Windows.

\section{Results}

Nineteen patients fulfilled the inclusion criteria; the parents of three patients refused participation because of a lack of time. The remaining 16 patients and 17 healthy controls entered the study. Characteristics of the participants are summarized in Table 1. There was no difference in BMI z score at the time of study between the patients who participated and the patients who refused participation. As keeping the dietary diaries and the activity reports appeared to be very time-consuming, not all parents managed to complete the program fully. The parents of five patients filled in the dairies during one 2-day period on DEXA instead of two 2-day periods and one 2-day period offDEXA instead of two 2-day periods. Parents of seven healthy controls filled in the dairies during 2 or 3 days instead of four. Mean energy intake and physical activity were calculated for days with complete registration.

\section{BMI and BMI z score}

In patients, mean BMI and mean BMI z scores at the time of the study were higher than at diagnosis. Mean increase in BMI $z$ score was $1.4 \pm 1.1$. Mean BMI z score at the time of the study was higher in patients compared to controls (Table 1).

Energy intake and physical activity in patients:

"on steroids" vs "off steroids"

In patients, mean energy intake on DEXA was higher than mean energy intake off DEXA. On the other hand, mean physical activity, measured by proxy report as well as by pedometer, was lower on DEXA compared to off DEXA (Table 1).

Energy intake and physical activity: patients vs healthy controls

Mean energy intake in patients on DEXA was higher compared to that of healthy controls, but mean energy intake in patients off DEXA was lower compared to healthy controls (Table 1). Mean physical activity measured by proxy report and measured by pedometer was lower in patients on DEXA compared to healthy controls. Mean 
Table 1 Characteristics of patients $(n=16)$ and controls $(n=17)$

\begin{tabular}{|c|c|c|c|}
\hline & \multicolumn{2}{|l|}{ Patients } & \multirow[t]{2}{*}{ Controls } \\
\hline & On steroids & Off steroids & \\
\hline Median age (year) & $5.8(4.2-15.3)$ & & $7.1(4.0-16.7)$ \\
\hline Median age at diagnosis (year) & $4.3(2.4-14.3)$ & & - \\
\hline Median interval from diagnosis (year) & $1.7(0.4-2.2)$ & & - \\
\hline Boys $(n)$ & 9 & & 8 \\
\hline Girls $(n)$ & 7 & & 9 \\
\hline Treatment regimen $(n)$ & & & - \\
\hline ALL-9 & 9 & & \\
\hline ALL-10 SR & 2 & & \\
\hline ALL-10 MR & 3 & & \\
\hline ALL-relapse & 2 & & \\
\hline Mean BMI $\left(\mathrm{kg} / \mathrm{m}^{2}\right)$ & $18.4 \pm 3.0$ & & $17.3 \pm 3.7$ \\
\hline Mean BMI z score & $1.1 \pm 1.2$ & & $0.2 \pm 1.1^{*}$ \\
\hline Mean BMI at diagnosis $\left(\mathrm{kg} / \mathrm{m}^{2}\right)$ & $15.6 \pm 1.6^{* *}$ & & - \\
\hline Mean BMI z score at diagnosis & $-0.4 \pm 0.9 * *$ & & - \\
\hline Mean change in BMI z score & $1.4 \pm 1.1$ & & - \\
\hline Mean energy intake $(\mathrm{kcal} / 24 \mathrm{~h})$ & $2,125.9 \pm 476.0 * * * * *$ & $1,305.0 \pm 249.4 * * * *, * * * * *$ & $1,775.1 \pm 426.1$ \\
\hline Mean physical activity ( $\mathrm{kcal} \mathrm{kg}^{-1} 24 \mathrm{~h}^{-1}$ ) & $30.0 \pm 3.9 * * * * * *$ & $38.8 \pm 6.1 * * * *$ & $40.0 \pm 6.0$ \\
\hline Mean physical activity (steps/24 h) & $7,303.1 \pm 4,622.9 * * * * * *$ & $11,376.7 \pm 5,471.8^{* * *}$ & $13,927.2 \pm 3,822.7$ \\
\hline
\end{tabular}

$A L L$ acute lymphoblastic leukemia, $S R$ standard risk, $M R$ medium risk, $B M I$ body mass index

${ }^{*} p<0.05$ vs patients at the time of study; ${ }^{* *} p<0.001$ vs patients at the time of study; ${ }^{* * *} p<0.05$ vs patients on steroids; $* * * *<<0.001$ vs patients on steroids; $* * * * * p<0.05$ vs controls (adjusted for age and gender); $* * * * * p<0.001$ vs controls (adjusted for age and gender)

physical activity measured by both proxy reports and pedometer in patients off DEXA was not significantly different compared to mean physical activity in healthy controls.

\section{Discussion}

We found increased energy intake and decreased physical activity in children with ALL during treatment with DEXA compared to energy intake and physical activity during treatment without DEXA. Energy intake on DEXA was higher compared to energy intake in healthy controls. Energy intake off DEXA was lower compared to energy intake in healthy controls. Physical activity in ALL patients on DEXA was lower than physical activity in healthy controls, whereas physical activity off DEXA was not significantly different from physical activity in healthy controls. This is in accordance with the clinical observation that many ALL patients are inactive and feel depressed while on steroids. The increase in BMI z score in patients between diagnosis and the time of the study confirms the clinical observation of weight gain in ALL patients on treatment with intermittent DEXA. Our study suggests that this weight gain might be the result of increased energy intake in combination with decreased physical activity during DEXA.

Energy intake during the maintenance phase of childhood ALL treatment has been studied scarcely. Reilly et al. found an increased energy intake in ALL patients during treatment with glucocorticoids [9]. However, they did not compare energy intake data in patients to those in healthy children. Bond et al. measured energy intake in ALL patients, just before a monthly vincristine and prednisone course. They found no differences in energy intake between ALL patients and healthy controls [4].

We found a decreased physical activity during DEXA courses compared to physical activity in healthy controls. These results are in accordance with those of Aznar et al., who also found a lower physical activity in ALL patients compared to healthy controls [2]. As far as we know, there are no further studies on physical activity measured in ALL patients on treatment. Most studies on physical activity in ALL patients were performed after completion of therapy $[8,13]$.

Our study has some limitations. The number of patients was only small. However, despite the small number, our results are supported by statistical analysis. All parents were highly motivated to participate in the study as they all recognized the problem of ongoing weight gain. Nevertheless, it appeared to be quite difficult to complete all the diaries, resulting in missing data. Finally, we did not evaluate the possible confounding effects of the cytostatic drugs that also form part of ALL treatment protocols.

Summarizing, we found an increased energy intake and a decreased physical activity in ALL patients during treatment with DEXA. The combination of high energy intake and low physical activity may contribute to the weight gain observed in childhood ALL patients during treatment. Therefore, 
physical activity should be encouraged, especially during treatment with DEXA. Further studies, to confirm our findings and to evaluate the effect of physical activity stimulation on obesity in children with ALL, are warranted.

Open Access This article is distributed under the terms of the Creative Commons Attribution Noncommercial License which permits any noncommercial use, distribution, and reproduction in any medium, provided the original author(s) and source are credited.

\section{References}

1. Ainsworth BE, Haskell WL, Whitt MC, Irwin ML, Swartz AM, Strath SJ, O'Brien WL, Bassett DR Jr, Schmitz KH, Emplaincourt PO, Jacobs DR Jr, Leon AS (2000) Compendium of physical activities: an update of activity codes and MET intensities. Med Sci Sports Exerc 32(Suppl 9):S498-S504 doi:10.1097/00005768200009001-00009

2. Aznar S, Webster AL, San Juan AF, Chamorro-Vina C, MateMunoz JL, Moral S, Perez M, Garcia-Castro J, Ramirez M, Madero L, Lucia A (2006) Physical activity during treatment in children with leukemia: a pilot study. Appl Physiol Nutr Metab 31 (4):407-413 doi:10.1139/H06-014

3. Beets MW, Patton MM, Edwards S (2005) The accuracy of pedometer steps and time during walking in children. Med Sci Sports Exerc 37 (3):513-520 doi:10.1249/01.MSS.0000155395.49960.31

4. Bond SA, Han AM, Wootton SA, Kohler JA (1992) Energy intake and basal metabolic rate during maintenance chemotherapy. Arch Dis Child 67(2):229-232

5. Davies JH, Evans BA, Jones E, Evans WD, Jenney ME, Gregory JW (2004) Osteopenia, excess adiposity and hyper- leptinaemia during 2 years of treatment for childhood acute lymphoblastic leukaemia without cranial irradiation. Clin Endocrinol (Oxf) 60(3):358-365 doi:10.1111/j.13652265.2003.01986.x

6. Fredriks AM, van BS, Wit JM, Verloove-Vanhorick SP (2000) Body index measurements in 1996-7 compared with 1980. Arch Dis Child 82(2):107-112 doi:10.1136/adc.82.2.107

7. Jarfelt M, Lannering B, Bosaeus I, Johannsson G, Bjarnason R (2005) Body composition in young adult survivors of childhood acute lymphoblastic leukaemia. Eur J Endocrinol 153(1):81-89 doi:10.1530/eje.1.01931

8. Mayer EI, Reuter M, Dopfer RE, Ranke MB (2000) Energy expenditure, energy intake and prevalence of obesity after therapy for acute lymphoblastic leukemia during childhood. Horm Res 53 (4):193-199 doi:10.1159/000023566

9. Reilly JJ, Brougham M, Montgomery C, Richardson F, Kelly A, Gibson BE (2001) Effect of glucocorticoid therapy on energy intake in children treated for acute lymphoblastic leukemia. J Clin Endocrinol Metab 86(8):3742-3745 doi:10.1210/ jc. 86.8 .3742

10. Reilly JJ, Ventham JC, Ralston JM, Donaldson M, Gibson B (1998) Reduced energy expenditure in preobese children treated for acute lymphoblastic leukemia. Pediatr Res 44(4):557-562 doi:10.1203/00006450-199810000-00015

11. Sainsbury CP, Newcombe RG, Hughes IA (1985) Weight gain and height velocity during prolonged first remission from acute lymphoblastic leukaemia. Arch Dis Child 60(9):832-836

12. Van Dongen-Melman JE, Hokken-Koelega AC, Hahlen K, De GA, Tromp CG, Egeler RM (1995) Obesity after successful treatment of acute lymphoblastic leukemia in childhood. Pediatr Res 38(1):86-90 doi:10.1203/00006450-199507000-00015

13. Warner JT, Bell W, Webb DK, Gregory JW (1998) Daily energy expenditure and physical activity in survivors of childhood malignancy. Pediatr Res 43(5):607-613 doi:10.1203/00006450199805000-00008 